\title{
Cognitive Policy Management Model for Ad Hoc Network
}

\author{
Yang Feng \\ Engineering \\ Hechi University \\ Yizhou, Guangxi, China \\ oyfo@163.com \\ Lan Chun-liang* \\ Hechi University \\ Yizhou, Guangxi, China \\ 1c17413@163.com \\ *Corresponding author
}

School of Physics and Mechanical \& Electrical

\author{
Liu Di \\ School of Physics and Mechanical \& Electrical \\ Engineering \\ Hechi University \\ Yizhou, Guangxi, China \\ liudi611@126.com \\ Song Hua-ning \\ School of Physics and Mechanical \& Electrical \\ Engineering \\ Hechi University \\ Yizhou, Guangxi, China \\ shn_2008@163.com
}

\begin{abstract}
The capabilities of self-aware and adaptive are not quickly and accurately enough, in Ad Hoc network. To dealing with those problems, we constructed a node hierarchy of context knowledge firstly, and then balance the loads to various nodes through the establishment of a distributed policy repository, so as to maintain the consistency of management strategies and maintain low overhead. Divide the cognitive module of context into four levels such as CKR, CIMT, CKDP and CKCP and then integrate with the distributed policy management module, finally, we presented a hierar-chical distribute policy management model based on collaborative context of cognitive mechanisms. The model can provide reference and support for the dynamic adaptive clustering algorithm design of Ad Hoc Network.
\end{abstract}

Keywords-Cognitive Policy; Distributed policy; Management Model; Hierarchical collaborative; Family networking; Ad Hoc network

\section{INTRODUCTION}

With the sustained development of computer technology, especially computer network technology, communication and network technology are continuing changing the way of communicate and lifestyle. In which, as an important branch of communications technology, the wireless communication technology has gradually stand out from the traditional communication technologies, the wireless communication technology could achieve the ideal personal communications goals that is anyone could exchange any kind of information at any time, any place with any people ${ }^{[1]}$.

In order to ensure the normal operation of the entire wireless communication network, the traditional wireless communication technologies need the base stations and other network infrastructure, this dependence on the underlying network facilities has become a hindrance to the development of wireless communication technology. In recent years, the new architecture of wireless communication network has been emerged and Ad Hoc network is the typical representative. Ad Hoc network is a non-central and multi-hop self-organizing wireless network $^{[2,3]}$, this archi-tecture is more flexible than the typical wireless communication network, behavior activities such as network transmis-sion, maintenance and management et al, are lack centralized equipment to control and manage ${ }^{[4]}$.

Although the Ad Hoc networks is a decentralized and self-organizing network, however, its current selfperception and adaptive capacity to the network environment are not quickly and accurately enough. Simultaneously, its maintainability is not ideal enough, the total cost is higher and the end to end transmission capacity is also not strong enough, therefore, traditional Ad Hoc network performance is need to be optimizing and improving. It is currently a hot academic research problem that introduce the cognitive mechanism into Ad Hoc Networks, research how to make it self-aware, how to make positive feedback and the right decisions for the perceived real-time status of each node ${ }^{[5,6]}$. These studies would help to improve the maintenance efficiency and network performance of Ad Hoc Networks.

\section{CONSTRUCTION AND FORMAL DESCRIPTION OF THE CONTEXT KNOWLEDGE MODEL}

In the strategic management model of $\mathrm{Ad} \mathrm{Hoc}$ Networks, the achievement of cognitive mechanisms need to use the "context" as a carrier of knowledge transfer between nodes. Therefore, the context is very important to the knowledge interaction, strategy activities execution and network performance optimization. The concept of context was first proposed by Schilit and Theimer ${ }^{[7]}$, they thought the context is the mark of people and things in the physical environment and their changes. Anind Dey ${ }^{[8]}$ has given a more common definition, he thought: context is all the entities status information which able to describe the the interaction between the user and the application , it include 
people, locations, objects, users, and the application itself. Specific to the Ad Hoc Network, its context includes several important informations such as nodes spatial information, campaign time information, nodes environmental information and nodes status information et al ${ }^{[9]}$.

In order to achieve the following goals such as monitor the status of Ad Hoc Networks and the effect of policy enforcement, as well as the summary, interpretation, analysis, inference and adaptive learning of the detected various information, we firstly structured the context knowledge model, its also the theoretical basis for subsequent operations. Common context knowledge model generally includes the following five kinds: key-valuepairs model, map model, object-oriented model, logicbased model and mark allocation model. In the above models, the mark allocation model is more suitable to construct the context knowledge model of the Ad Hoc network, because it use the hierarchical structure markup language such as XML and RDF to express the context knowledge. First, organize the Ad Hoc network nodes according to a hierarchical data structures, and it can be used to construct the context knowledge. The hierarchical structure of node's context knowledge is shown in fig. 1 .

Context knowledge of each node mainly includes: node position, remaining energy, running state, transmission capability, functional characteristics and head cluster properties et al. We collect the node' accuracy \& latitude to marke the position information, and use its movement direction \& speed to express the running status for the movement nodes, and use the available bandwidth \& data transfer rate to measure its transfer ability, and count the two indicators of its different features; These method can help to building the policy management model. For the efficiency of collection \& interpretation \& summary \& reasoning $\&$ analysis the context knowedge through the cognitive node, we build a XML file according to the unified form of structure. The specific circumstance is shown in Fig .2.

\section{DESIGN \& EXPRESS OF POLICY MANAGEMENT MODEL BAED ON CONTEXT OF COGNITIVE MECHANISMS}

\section{A. Distribute Policy-Based Management Model}

Policy-Based Management method can help the managed system to automatically and dynamically adjust its behaviors through the policy-driven management, and it has good adaptive characteristics. The traditional strategic management model is a typical network-centric concept, it deviates from these features that the Ad Hoc network is lack of infrastructure support and battery-powered. Inorder to improve the scalability of PBM, we need to do the distributing process. Balance the load to each node of the network, and keep the maintain consistency of the management strategies, and try to maintain a low overhead. In this paper, we introduced a Distribute Policy Repository and divided all the nodes into three roles ${ }^{[10]}$ : Distributed Manager Center, Cluster Head and Cluster Leaguer, the specific structure is shown in Fig.3.

We divide all nodes into two types of modules according to the functional requirements, they are Cluster Module and Terminal Module. The role \& function of PMT \& DPR \& PDP and PEP are shown in table 1.
TABLE I. MAPPING TABLE OF ROLES AND FUNCTIONS

\begin{tabular}{ccc}
\hline PBM & role & Function(policy) \\
\hline PMT & DMC & Propose \& edit \\
DPR & DMC & Storage, distribute \\
PDP & DMC, CH & Monitor, making-decision \\
PEP & DMC $, \mathrm{CH}, \mathrm{CL}$ & Implement, report \\
\hline
\end{tabular}

B. Mechanism Construction of Context Recognition

Inorder to make the Distribute Policy-Based Management Model has the ability of context-aware, we introduced the context cognitive module, includeing Context Knowledge Repository \& Context Intelligence Management Tool \& Context Knowledge Decision Point and Context Knowledge Collection Point ${ }^{11,12]}$; as shown in Fig. 4.

By using the context cognitive module and PBM, we proposed a new policy management model shown in fig. 5, it has hierarchical collaborative and context of cognitive mechanisms.

In which, the solid line represents the policy data transfer direction and the dotted line represents the transfer direction of the context knowledge. Its transmission process is divided into the following steps:

Step. 1 CKDP explain \& summary the context knowledge after CKCP has collected and transferred it;

Step.2 Transfer the explicit context knowledge to the same layer PDP and the up layer CIMT;

Step.3 Transfer the tacit context knowledge to the same layer PMT and store in CKR, after the CIMT rea-soning \& analysis and learning it;

The PMT \& CIMT in the management layer adjust the management strategies and the cluster elements' statement, according to the QoS demands. After completing the selflearning of knowledge and the adaptive process of network, we can achieve the purpose of optimizing the QoS performance.

\section{EPILOGUE}

In this paper, we constructed a context knowedge model and formal described it, by introducing the context of cognitive mechanisms into the network's strategic management model building process. And then, we balanced the loads to various nodes through the establishment of a distributed policy repository, so as to maintain the consistency of management strategies and maintain low overhead. Finally, we proposed a new policy management model for Ad Hoc network. The model can implement the context of cognitive mechanisms and can joint the four layers of Execution Layer \& Decisionmaking Layer \& Data Layer \& Management Layer. In the future work, we need to research the dynamic adaptive clustering network algorithm to further meet the QoS demands.

\section{ACKNOWLEDGMENT}

This project was supported by Research projects of hechi university(2013ZX-N004;2013A-N004) 


\section{REFERENCES}

[1] David J. Wireless Personal Communications [R].Wesley: Addison Longman Incorporated, 2007.

[2] Kim, Seungmo. The capacity of cognitive ad hoc networks [C]. //16th Asia-Pacific Conference on Communications, 2010:p395400.

[3] Hieu C,Hong C. Securely Deliver Data by Multi-path Routing Scheme in Wireless Mesh Networks[C]. //Computational Sci-enceICCS, 2007: 475- 481 .

[4] JUBIN J, TORNOW J. D. The DARPA Packet Radio Network Protocols[C]. // Proceedings of the IEEE, 2007: p21-32.

[5] Su, Weifeng. Active cooperation between primary users and cognitive radio users in cognitive ad-hoc networks [C]. // Proceedings of the International Conference on Acoustics, 2010:p3174-3177

[6] Jian-zhao, Zhang. A fast neighbor discovery algorithm for cognitive radio ad hoc networks[C]. // 12th International Conference on Communication Technology (ICCT'2010), 2010:p446-449.
[7] Brown, Chen X. Context-Aware. Applications: from the Laboratory to the Marketplace [J].IEEE Communications, 2007, 21(5):p58-64

[8] P. Sutton, L. E. Doyle, K. E. Nolan. A reconfigurable platform for cognitive networks[C]. // Proc of CROWNCOM 2006, 2006:332340

[9] XIONG Jian, LI Yuan, YANG Zhen-hai. A NEW PARAMETRIC TEST FOR TIME SERIES [J]. JOURNAL OF SOUTH CHI-NA NORMAL UNIVERSITY (NATURAL SCIENCE EDITION), 2008,03:5-8.

[10] ZHANG Long, ZHOU Xian-Wei, WANG Jian-Ping, et al. Routing Protocols for Delay and Disruption Tolerant Networks[J]. Journal of Software, 2010,21(10):2554-2572.

[11] Li Yong, Jiang Yurong, Jin Depeng, et al. "Energy-efficient optimal opportunistic forwarding for delay-tolerant networks". IEEE Transactions on Vehicular Technology, 2010, 59(9):45004512 .

[12] YANG Hui-zhong, ZANG Yong. Comparisons of bias compensation methods and other identification approaches for Box-Jenkins models [J]. Control Theory \& Applications, 2007, 24(02):215-222

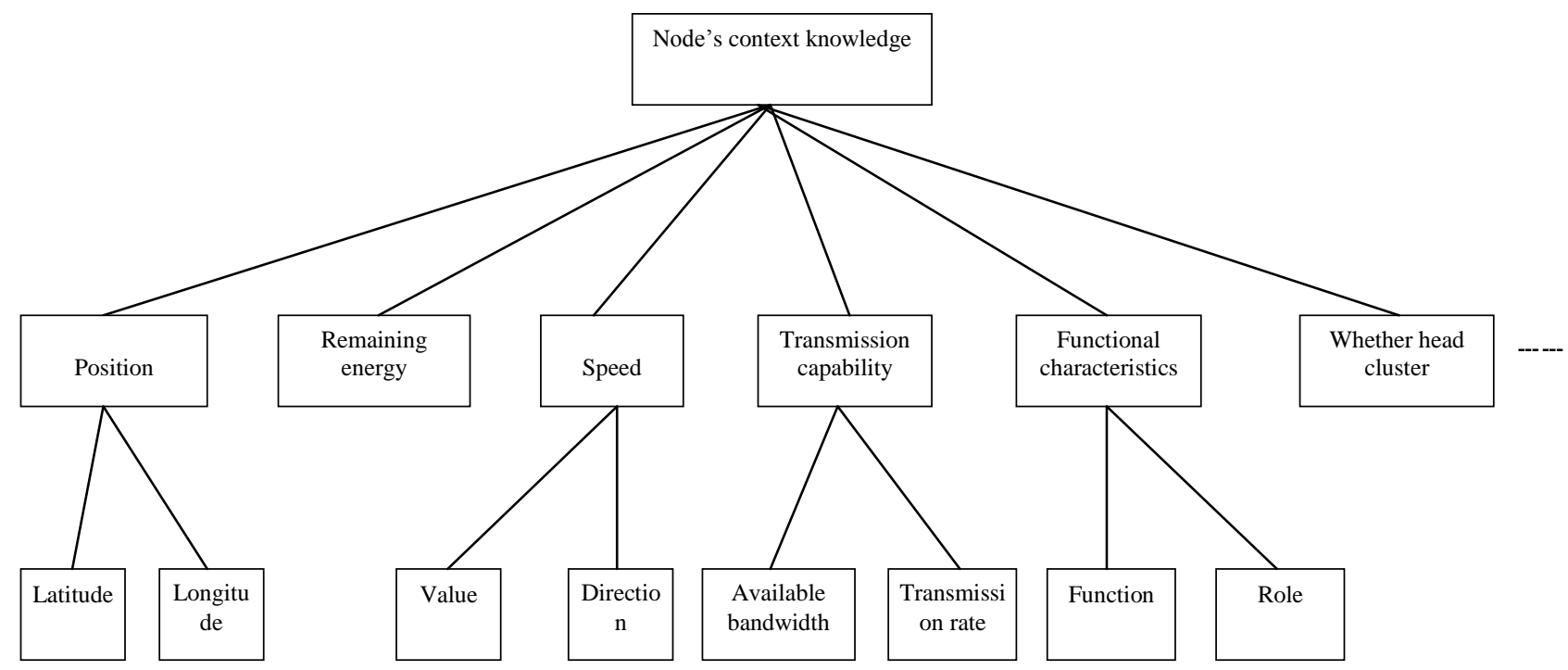

Figure 1. hierarchical structure of node's context knowledge

$<$ ?xml version=" 1.0 " encoding $="$ utf- 8 "? $>$

<xs:schema xmlns:xs="http://www.w3.org/2001/XMLSchema-

instance"

elementForDeault="qualified"attributeFormDefault="unqualified">

$\langle x s: c o m p l e x$ Type name $="$ nodes context knowledge ">

$<x s:$ attribute name $="$ Position">

$\langle$ xs:attribute name $="$ Speed">

$\langle x s:$ attribute name=" Remaining energy" $>$

$\langle x s$ :attribute name $="$ Transmission capability" $>$

$<x s:$ attribute name=" Functional characteristics">

$\langle x s$ attribute name $="$ Whether head cluster" $>$

$<$ xs:complex Type $>$

$</ x s$ :schema $>$

Figure 2. XML description of context knowledge 


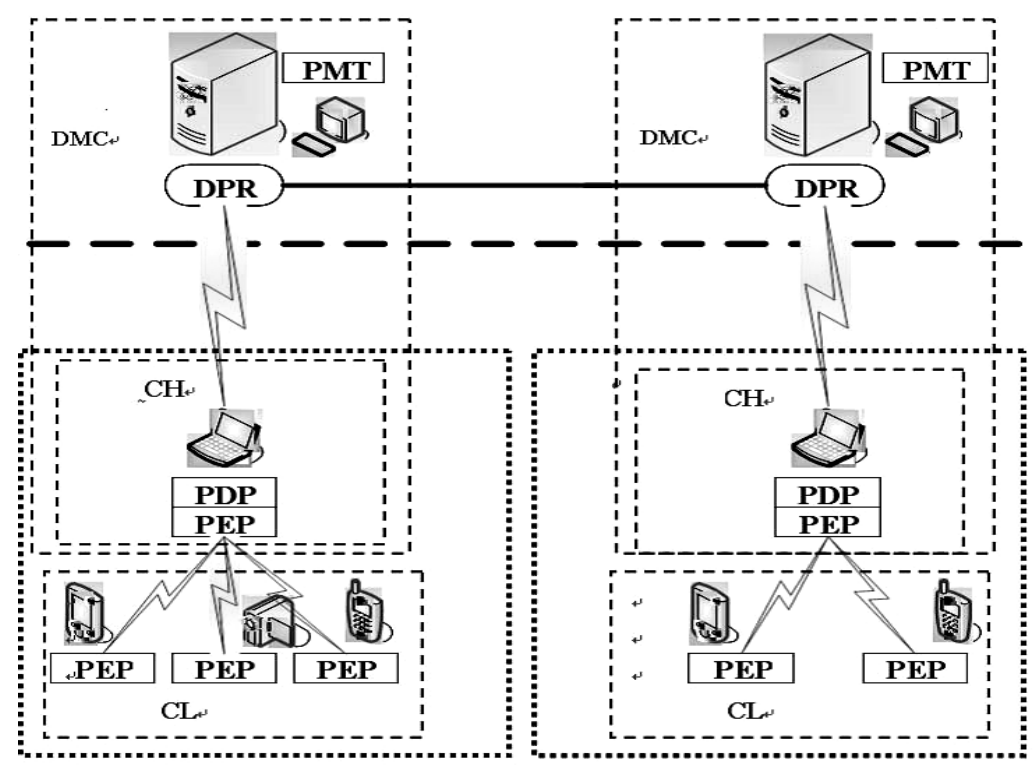

Figure 3. Schematic of roles \& clusters division for Distribute Policy-Based Management Model
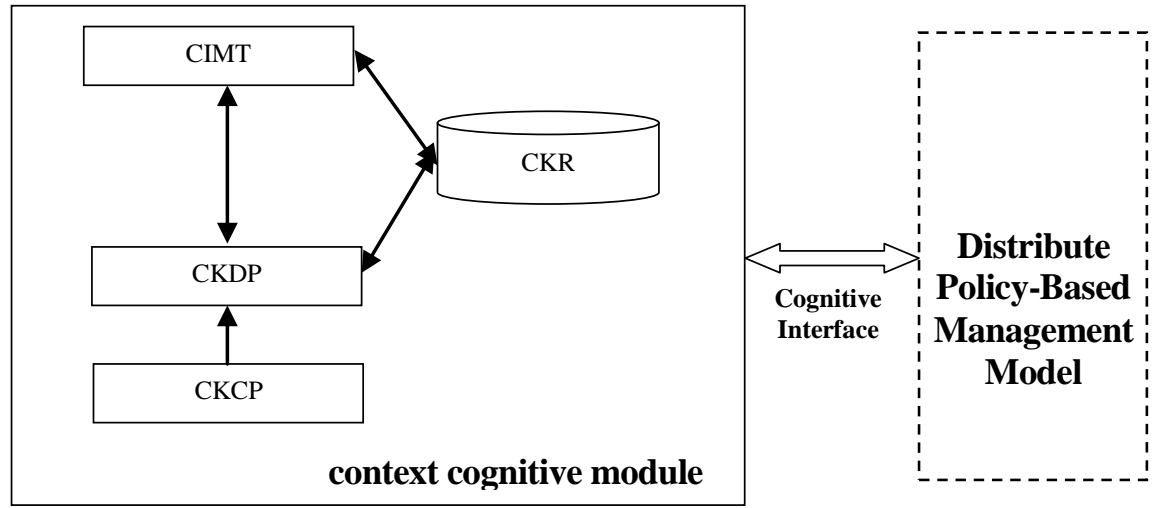

Figure 4. Four-element assembly of context cognitive module

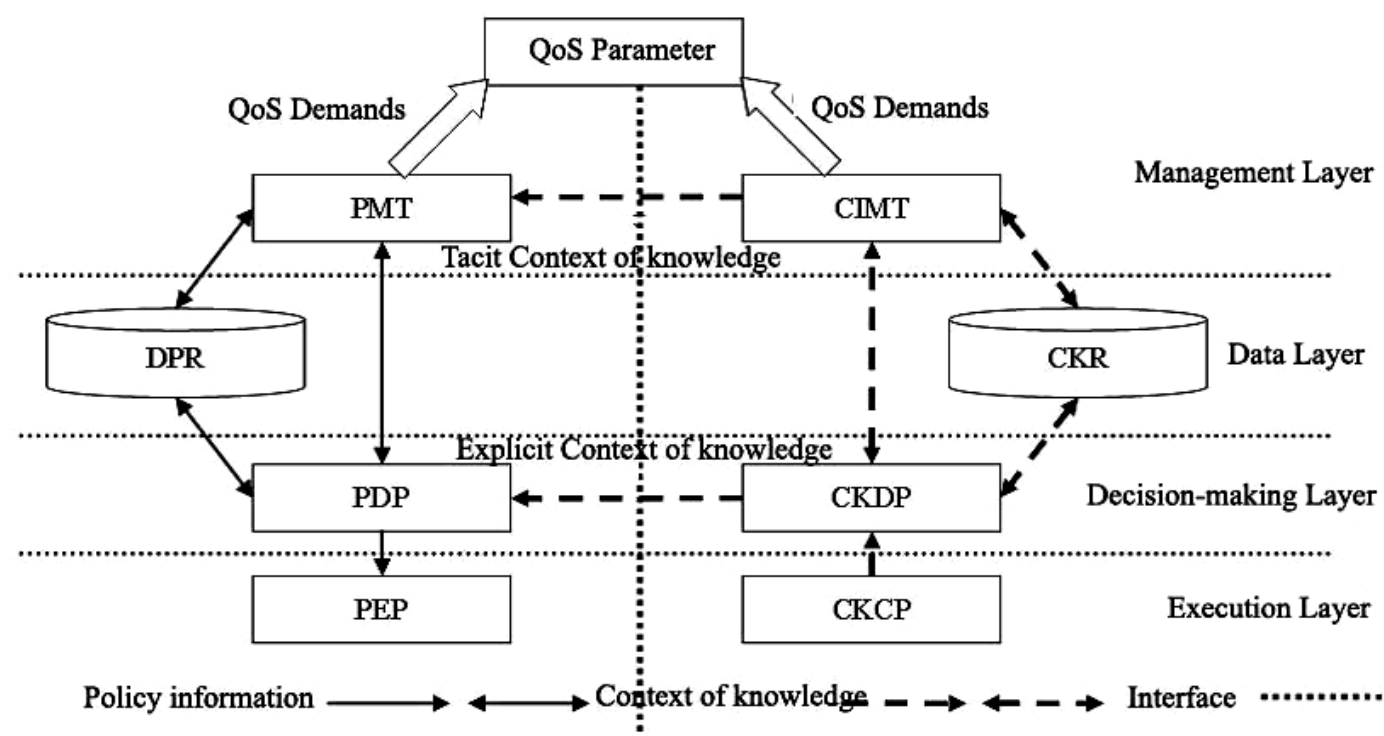

Figure 5. Policy management model 